\title{
Low energy catheter ablation of right ventricular outflow tract tachycardia
}

\author{
Arif J Ahsan, David Cunningham, Edward Rowland
}

\begin{abstract}
A 38 year old woman with a structurally normal heart presented with near syncope and had right ventricular outflow tract tachycardia. She was intolerant of antiarrhythmic medication and underwent low energy catheter ablation. Six non-arcing shocks of $25 \mathrm{~J}$ were delivered to the right ventricular outflow tract. No further ventricular tachycardia occurred during a follow up of seven months without antiarrhythmic treatment.
\end{abstract}

Ventricular tachycardia of left bundle branch configuration and with an inferior axis in patients with structurally normal hearts is recognised as a clinical syndrome. ${ }^{1-3}$ It is usually brought on by stress and exercise and normally has a good prognosis. ${ }^{1}$ Unlike most ventricular tachycardia, it can be suppressed by treatment with $\beta$ blockers and calcium channel blockers. These features are consistent with a catecholamine sensitive focus arising from the right ventricular outflow tract. Experience with surgery and catheter ablation in this condition is limited.

We describe a patient with right ventricular outflow tract tachycardia and a structurally normal heart who was intolerant of antiarrhythmic medication and in whom low energy catheter ablation was successful.

\section{Case report}

CLINICAL PRESENTATION

A 38 year old woman was referred because of two episodes of near syncope that occurred on exertion. She had also experienced several episodes of dizziness lasting a few seconds, without noticeable palpitation. She complained of dyspnoea but she could climb 20 stairs. She smoked 20 cigarettes per day. Clinical examination was entirely normal. The electrocardiogram at rest and chest $x$ ray were normal. Echocardiography showed normal chamber sizes and normal valves. Radionuclide ventriculography at rest was normal for both ventricles. Magnetic resonance imaging showed normal left and right ventricular dimensions, wall thickness, wall thickening, and ejection fractions.

She performed the modified Bruce protocol on a treadmill. Before she mounted the treadmill her heart rate increased to 100 beats/ minute and she developed frequent unifocal ventricular extrasystoles with a left bundle branch configuration and inferior axis (fig 1). After two minutes and 45 seconds of stage I she developed frequent bouts of nonsustained ventricular tachycardia (cycle length
Royal Brompton National Heart and Lung Hospital, London A J Ahsan

D Cunningham

E Rowland

Correspondence to Dr Arif J Ahsan, Department of Pacing and Electrophysiology, Royal Brompton physiology, Royal Brompton National Heart and Lung Hospital, Sydney Stree

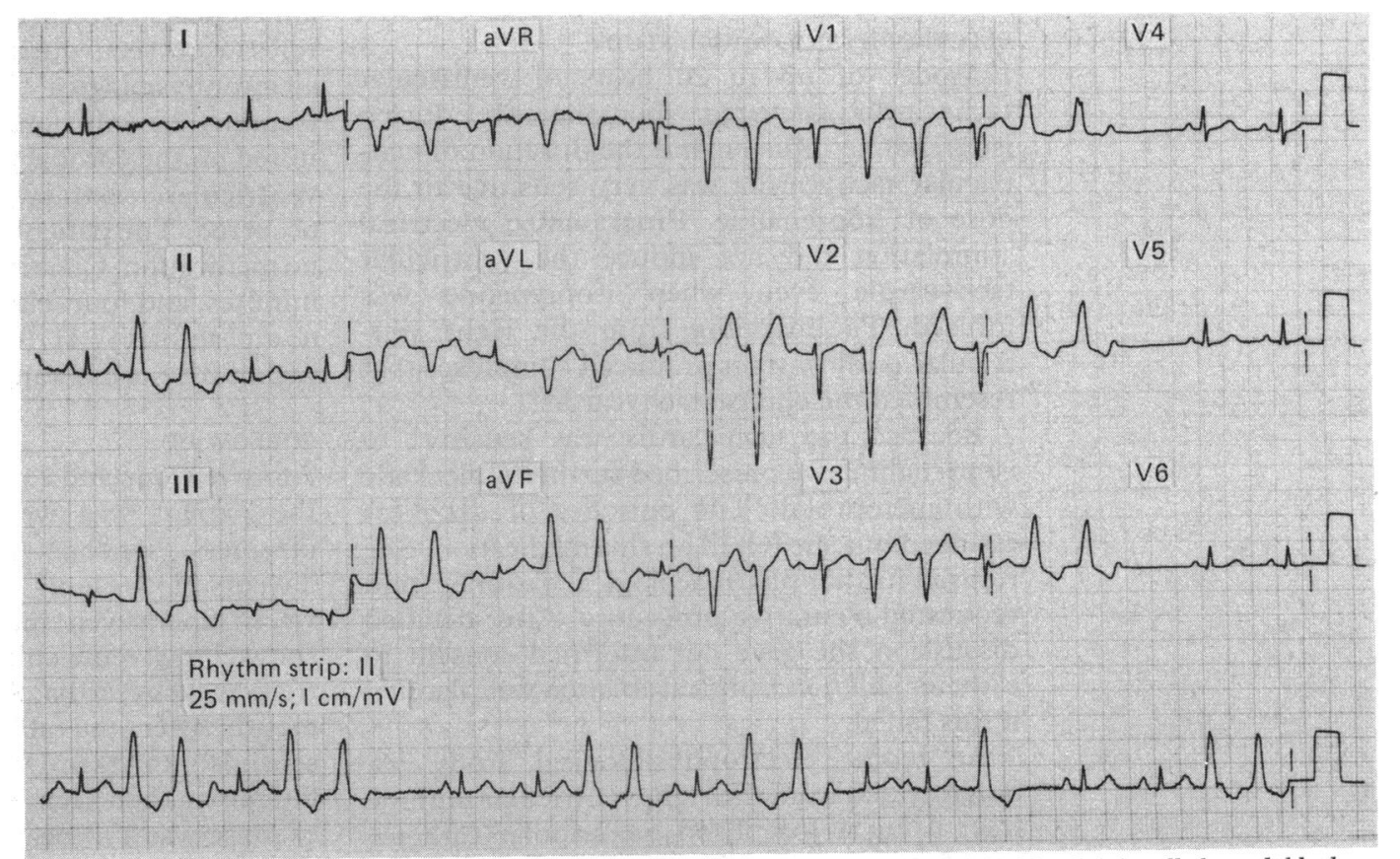

Figure 1 Twelve lead electrocardiogram showing frequent ventricular extrasystoles with a left bundle branch block and inferior axis configuration. 


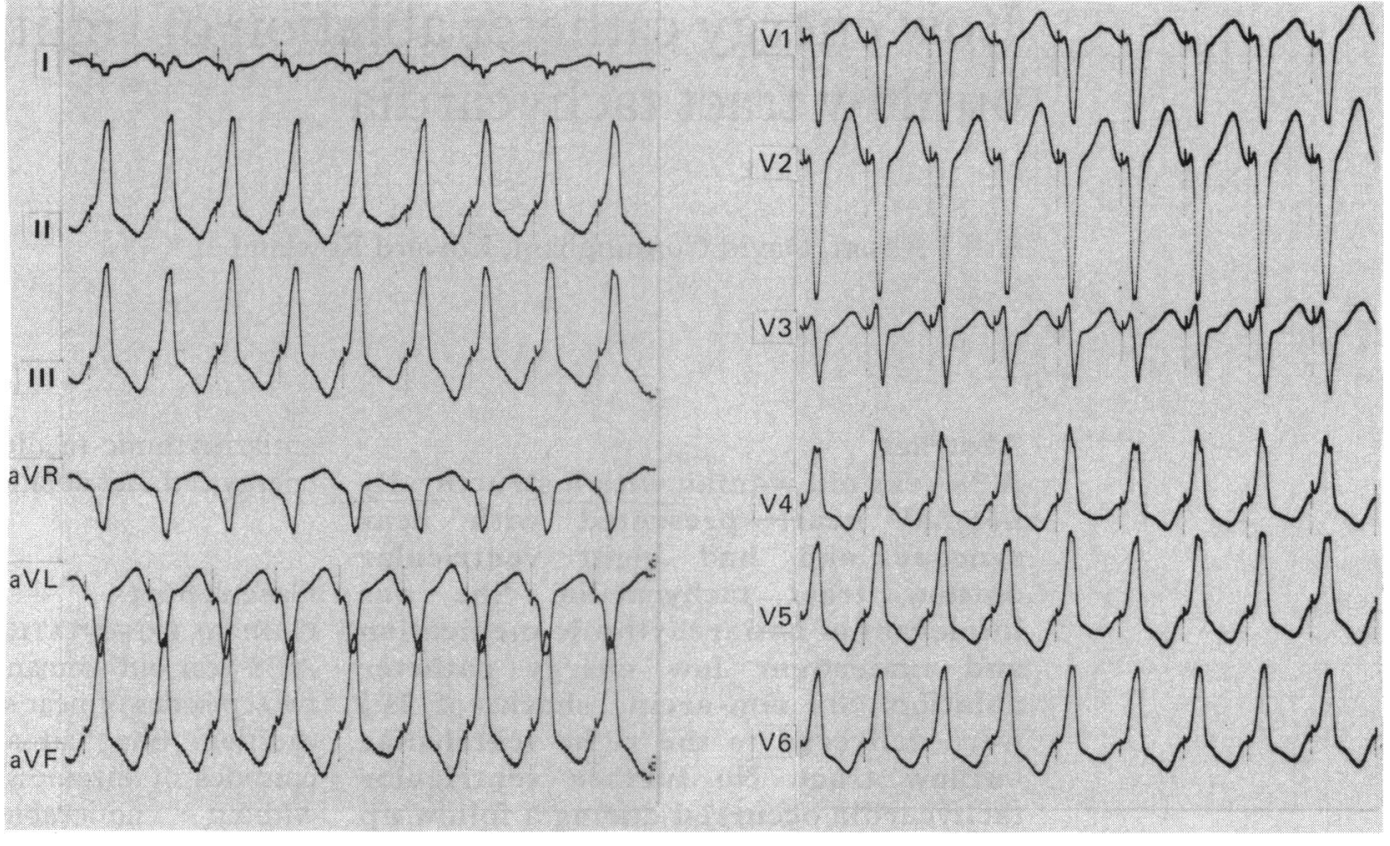

Figure 2 Twelve lead electrocardiogram produced by pacing at the ablation site. The configuration of the $Q R S$ complexes closely matched that of the ventricular extrasystoles in fig 1.

$380 \mathrm{~ms}$ ) with the same configuration as the previous extrasystoles. She felt unwell and the test was stopped. The episodes of ventricular tachycardia rapidly ceased but she continued to have single ventricular extrasystoles for six minutes. After her admission to hospital a repeat exercise test was performed. Again she developed frequent unifocal extrasystoles with a similar configuration before she mounted the treadmill. This time she exercised for 55 seconds into stage III before sustained ventricular tachycardia developed (cycle length $380 \mathrm{~ms}$ ). She was given intravenous lignocaine and after 20 seconds the rhythm reverted to sinus tachycardia. She was subsequently treated with sotalol but was intolerant of this.

\section{ELECTROPHYSIOLOGICAL STUDY}

Episodes of up to 20 beats of ventricular tachycardia occurred spontaneously during isoprenaline infusion and the duration of ventricular tachycardia was very sensitive to the dose of isoprenaline. Programmed electrical stimulation did not induce the ventricular tachycardia even when isoprenaline was infused. Pacemapping from the right ventricular outflow tract produced complexes that resembled the clinical tachycardia.

Because the tachycardia was sensitive to isoprenaline, we prescribed further $\beta$ blockade with metoprolol. The episodes of dizziness resolved but she felt ill on this medication. She refused further pharmacological treatment and requested a curative procedure. After detailed discussion she gave her informed consent to catheter ablation; surgical ablation was planned if this failed.

At repeat electrophysiological study we pacemapped from the right ventricular outflow tract using a Josephson quadripolar catheter with $5 \mathrm{~mm}$ spacing. We manipulated the catheter until we obtained the best pacemap (fig 2).
A contoured ablation electrode ${ }^{4}$ was placed at this site. We used a previously described protocol $^{5}$ to deliver six shocks of $25 \mathrm{~J}$ to both the proximal and distal poles of the ablation catheter at this site with a $\mathrm{NHH}$ ablater ${ }^{4}$ as the power source and a chest paddle over the left side of the sternum as the cathode. The arrhythmia that followed the delivery of the shocks was transient: ventricular tachycardia lasting five to 15 seconds followed the delivery of shocks three to six. It had a left bundle branch configuration but the frontal axis was different from that of the clinical ventricular tachycardia. This did not have any haemodynamic consequences. After three of the shocks there was transient atrial fibrillation with a controlled ventricular rate and no haemodynamic effects. No further ventricular extrasystoles were seen during the remainder of the electrophysiological study despite further isoprenaline infusion. The patient was monitored on the coronary care unit but no further ventricular extrasystoles or arrhythmias occurred. The patient was discharged home on no medication. Creatine kinase measured at five minutes, and four, eight, 18 , and 24 hours rose to a peak of $117 \mathrm{IU} / 1$ at eight hours, which is within the normal range for our laboratory.

\section{FOLLOW UP}

At review one and seven months after ablation the patient was well. She had no further dizziness, syncope, dyspnoea, or tiredness. Twenty four hour Holter monitoring two weeks and seven months after the ablation showed no ventricular extrasystoles or ventricular tachycardia. At a repeat exercise test a month after the ablation she exercised 10 seconds into stage IV before being limited by fatigue in her legs. Her heart rate at peak exercise was 170 beats/minute and there were no ventricular extrasystoles or ventricular tachycardia. 


\section{Discussion}

The ventricular tachycardia in this patient was uncharacteristic of that seen with ischaemic heart disease. It was brought on by exercise, stress, and isoprenaline infusion and extrasystoles were not recorded if the heart rate was below 100 beats/minute. It was abolished by metoprolol. This is all consistent with the tachycardia being very sensitive to catecholamine concentration. It was not inducible at electrophysiological study, even when isoprenaline was infused. The failure to induce the tachycardia with programmed electrical stimulation suggests that it was caused by automaticity or triggered activity not reentry.

The site of origin was localised by pacemapping to the right ventricular outflow tract. This is consistent with other reports. ${ }^{126} \mathrm{We}$ used an ablation system designed to reduce the delivered energy and barotrauma. ${ }^{4}$ We believe that many of the serious side effects seen with conventional ablation are caused by the high energies used and the accompanying large pressure disturbances. These side effects have limited the use of ablation and a recent report of the world registry concluded that the use of ablation in the ventricle remains experimental. ${ }^{7}$ Measurements of voltage and current during the delivery of these six shocks showed that impedance remained constant and indicated that arcing had not taken place and therefore that no pressure disturbance had occurred. ${ }^{8} \mathrm{We}$ suggest that it is particularly important to avoid barotrauma in thin walled structures such as the right ventricle and coronary sinus because of an increased risk of rupture. We believe that the use of this low energy ablation system will improve safety and reduce complications. The lack of rise in serum creatine kinase is also consistent with reduced myocardial damage.

This is the first report of successful low energy catheter ablation of a catecholamine sensitive right ventricular outflow tract tachycardia. Reports of high energy catheter ablation in this condition are rare. Wilber et al described a patient in whom two conventional shocks of $200 \mathrm{~J}$ were delivered to a site in the outflow tract after an excellent pacemap but in whom ventricular tachycardia returned after several hours. This patient went on to have successful surgical ablation. ${ }^{6}$ At surgery the site of earliest endocardial breakthrough was $2 \mathrm{~cm}$ lateral to the site of the catheter ablation. Hartzler described, in the first report of catheter ablation for ventricular tachycardia, a patient with successful ablation of right ventricular outflow tract tachycardia; this followed unsuccessful surgical endocardial resection. ${ }^{9}$
In this patient we delivered six low energy shocks. Our previous animal work (unpublished) showed that increasing numbers of low energy non-arcing shocks can produce lesions of similar size to those produced by shocks of much higher energies but without causing dangerous large pressure disturbances. Thus we believe that it is safer to deliver repeated non-arcing shocks than single much larger shocks. Unfortunately, failure to induce the tachycardia immediately after the ablation does not predict success in the next few hours or days because the cells essential to the tachycardia mechanism may be reversibly damaged and not necrotic. Thus at present there is no reliable end point at which ablation can be stopped. In this case ventricular tachycardia was not inducible; but after the first three shocks we saw single ventricular extrasystoles with the same configuration as the clinical ventricular tachycardia. Because we thought that insufficient damage had been produced we delivered three further shocks. After each of these we saw no further extrasystoles with the configuration of ventricular tachycardia.

The report by Wilber et $a l^{6}$ and our report are consistent with this syndrome being caused by a relatively localised abnormal area rather than a diffuse myocardial process. The localised nature of the substrate suggests that this syndrome may be suitable for treatment by catheter ablation in patients intolerant of medication.

AJA was supported by a grant from the Clinical Research Committee, the National Heart and Chest Hospitals.

1 Buxton AE, Waxman HL, Marchlinski FE, et al. Righ ventricular tachycardia: clinical and electrophysiological characteristics. Circulation 1983;68:917-27.

2 Palileo EV, Ashley WW, Swiryn S, et al. Exercise provocable right ventricular outflow tract tachycardia. $A m$ Heart $J$ 1982;104:185-93.

$3 \mathrm{Wu} \mathrm{D}$, Kou H, Hung J. Exercise-triggered paroxysmal ventricular tachycardia: a repetitive rhythmic activity possibly related to afterdepolarisation. Ann Intern Med 1981;95:410-4.

4 Ahsan AJ, Cunningham AD, Rowland E, Rickards AF Catheter ablation without fulguration. Design and performance of a new system. PACE 1989;12:1557-61.

5 Rowland E, Cunningham D, Ahsan A, Rickards A. Transvenous ablation of atrioventricular conduction with a low energy power source. Br Heart J 1989;62:361-6.

6 Wilber DJ, Blakeman BM, Pifarre R, et al. Catecholamine sensitive right ventricular outflow tract tachycardia: intraoperative mapping and ablation of a free wall focus. $P A C E$ 1989;12:1851-6.

7 Evans GT, Scheinmann MM, Zipes DP, et al. The percutaneous cardiac mapping and ablation registry: summary of results. $P A C E$ 1988;11:1621-4.

8 Cunningham AD, Ahsan AJ, Rowland E, Rickards AF. Impedance changes during catheter ablation and their relationship to electrical arcing and clinical efficacy. $P A C E$ relationship to elec

9 Hartzler GO. Electrode catheter ablation of refractory focal ventricular tachycardia. J Am Coll Cardiol 1983;2: 1107-13. 